\title{
Sounds of instability from generalized QCD ghost dark energy
}

\author{
Esmaeil Ebrahimi ${ }^{1,2} *$ and Ahmad Sheykhi ${ }^{2,3} \dagger$ \\ 1 Department of Physics, Shahid Bahonar University, PO Box 76175, Kerman, Iran \\ 2 Research Institute for Astronomy and Astrophysics of Maragha (RIAAM), Maragha, Iran \\ ${ }^{3}$ Physics Department and Biruni Observatory, College of Sciences, Shiraz University, Shiraz 71454, Iran
}

\begin{abstract}
We investigate about the stability of generalized QCD ghost dark energy model against perturbations in the FRW background. For this purpose, we use the squared sound speed $v_{s}^{2}$ whose sign determines the stability of the model. We explore the stability of this model in the presence/absence of interaction between dark energy and dark matter in both flat and non-flat geometry. In all cases we find almost a same result. Based on the square sound speed analysis, due to the existence of a free parameter in this model, the model is theoretically capable to lead a dark energy dominated stable universe. However, observational constraints rule out such a chance. In conclusion, we find evidences that the generalized ghost dark energy might can not lead to a stable universe favored by observations at the present time.
\end{abstract}

\section{INTRODUCTION}

Seeking a convincing explanation for the present acceleration of the cosmic expansion [1], a variety of models have been proposed in the literature. These models should be consistent with observational evidences supporting the acceleration phase of the universe expansion 2 25]. All of the models can be categorized in two different groups the dark energy (DE) [6-15] and the modified gravity [16 19] models. Both of these approaches are extensively investigated in the literature during the past decade. In this paper we are interested in DE approach. DE models are based on the assumption that the correct theory of gravitation in large scale is the Einstein's general relativity (GR). In this approach it is assumed that there exist an exotic type of energy which its equation of state parameter is negative and push the universe to accelerate.

One main problem in solving the DE problem is introducing new degrees of freedom which may lead to inconsistencies. Thus avoiding such problem we are more interested in DE models, based on already presented degrees of freedom in physics. One interesting model of this category is the so called ghost dark energy (GDE) model proposed recently [20 22]. Seeking a solution to $U(1)$ problem, the so-called Veneziano ghost has been proposed in the low energy effective QCD where they are completely decoupled from the physical sector 23 26]. However, they contribute to the vacuum energy in curved space or time-dependent background. In 21] the authors discussed that this vacuum energy can play the role of DE. This contribution to the vacuum energy in curved space-time is proportional to $\Lambda_{Q C D}^{3} H$, where $H$ is the Hubble parameter and $\Lambda_{Q C D}^{3}$ is QCD mass scale. With $\Lambda_{\mathrm{QCD}} \sim 100 \mathrm{MeV}$ and $H \sim 10^{-33} \mathrm{eV}, \Lambda_{\mathrm{QCD}}^{3} H$ gives the right order of magnitude $\sim\left(3 \times 10^{-3} \mathrm{eV}\right)^{4}$ for the observed DE density [21]. Following this interesting coincidence, various aspects of GDE have been studied [27 35, 40].

In [37], the authors discussed that the contribution of the Veneziano QCD ghost field to the vacuum energy is not exactly of order $H$ and a subleading term $H^{2}$ appears due to the fact that the vacuum expectation value of the energy-momentum tensor is conserved in isolation [36]. They argued that the vacuum energy of the ghost field can be written as $H+O\left(H^{2}\right)$, where the subleading term $H^{2}$ in the GDE model might play a crucial role in the early evolution of the universe, acting as the early DE. Based on this idea people considered the role of this version of the Veneziano ghost field energy to the DE problem and tried to see if there exist a better agreement between this latter model and the observations. We call this model as generalized ghost dark energy (GGDE). In this model the energy density is written in the form $\rho_{D}=\alpha H+\beta H^{2}$, where $\beta$ is a constant [38]. It was shown [38] that taking the subleading term $H^{2}$ into account can give better agreement with observational data compared to the usual GDE. The GGDE has attracted a lot interest recently [39, 40]. It was shown [38, 39], that the subleading term $H^{2}$ in the energy density has negative contribution compared to the leading term $H$. This result first pointed out in [38] for flat FRW universe and then confirmed for non-flat universe [39].

Based on all mentioned above, in this paper we would like to investigate the effects of the subleading term on the background perturbations and stability of the GGDE model. It is worth mentioning that in [28], we found that the GDE is instable against perturbations and a stable DE dominated universe cannot be achieved in such a model.

\footnotetext{
* eebrahimi@uk.ac.ir

$\dagger$ asheykhi@shirazu.ac.ir
} 
From observations we know that our universe is in a DE dominated phase. Thus any viable DE model should result a stable DE dominated universe. One simple way to check such a stability for any new DE model is to discuss the behavior of the square sound speed $\left(v_{s}^{2}=d p / d \rho\right)$ [1] in a DE dominated universe. The sign of $v_{s}^{2}$ plays a crucial role in determining the stability of the background evolution. If $v_{s}^{2}<0$, it means that we have the classical instability of a given perturbation. In contrast $v_{s}^{2}>0$, leaves chance for greeting a stable universe against perturbations. However, this does not enough insight to say the model is surely stable but at least can show sounds of instability in the model. This approach has been used for exploring some DE models. For example in [42, 43] the authors investigated the behavior of the square sound speed for holographic DE as well as the agegraphic DE models and found both of these models are instable against background perturbations. Also it was shown that chaplygin gas and tachyon DE have positive squared speeds of sound with, $v_{s}^{2}=-w$, and thus they are supposed to be stable against small perturbations [44, 45]. The stability of the original GDE was studied in [28], and we found the GDE is not capable to result a stable DE dominated universe. The issue was investigated for flat, non-flat in the presence/absence of the interaction between DE and dark matter. Also, a same procedure was considered in [46] to show the stability of the GDE in the chameleon Brans-Dicke theory.

In this paper our aim is to discuss the chance of the GGDE model in resulting to DE dominated universe. In recent years several signals have been detected, implying a small interaction between DE and DM is possible. As an instance, observational evidences provided by the galaxy cluster Abell A586 supports the interaction between DE and DM [47]. Also evidences from CMB and also supernova measurements of the cubic correction to the luminosity distance favor a positively curved universe [48, 49]. Due to the above observational evidences we consider the stability of GGDE model in the presence of interaction in both flat and non-flat background.

This paper is organized as follows. In the next section, we review the GGDE model in flat universe and discuss its instability against perturbation. In section III we explore the stability of the GGDE model in the presence of the interaction term in flat universe. Section IV is devoted to stability of the GGDE model in a interacting, non-flat universe. We summarize our results in section $\mathrm{V}$.

\section{STABILITY OF NON-INTERACTING GGDE MODEL}

\section{A. Review of the non-interacting GGDE in flat universe}

At first we briefly discuss the non-interacting GGDE in a flat FRW background. For flat universe, the first Friedmann equation read

$$
H^{2}=\frac{8 \pi G}{3}\left(\rho_{m}+\rho_{D}\right)
$$

where $\rho_{m}$ and $\rho_{D}$ are, respectively, the energy densities of pressureless matter and DE. The dimensionless energy density parameters are defined as

$$
\Omega_{m}=\frac{8 \pi G \rho_{m}}{3 H^{2}}, \quad \Omega_{D}=\frac{8 \pi G \rho_{D}}{3 H^{2}}
$$

According to these definitions, the first Friedmann equation (11) can be rewritten as

$$
\Omega_{m}+\Omega_{D}=1 \text {. }
$$

The conservation equations also read

$$
\begin{aligned}
\dot{\rho}_{m}+3 H \rho_{m} & =0 \\
\dot{\rho}_{D}+3 H \rho_{D}\left(1+w_{D}\right) & =0
\end{aligned}
$$

which imply that matter and DE components are separately conserved.

The energy density of GGDE is defined as [38]

$$
\rho_{D}=\alpha H+\beta H^{2}
$$

where $\alpha$ is a constant of order $\Lambda_{\mathrm{QCD}}^{3}$ and $\Lambda_{\mathrm{QCD}}$ is QCD mass scale. In the original GDE with $\Lambda_{\mathrm{QCD}} \sim 100 M e V$ and $H \sim 10^{-33} \mathrm{eV}, \Lambda_{\mathrm{QCD}}^{3} H$ gives the right order of magnitude $\sim\left(3 \times 10^{-3} \mathrm{eV}\right)^{4}$ for the observed DE density [21]. In the GGDE, $\beta$ is a free parameter which can be adjusted for better agreement with observations. 
Taking the time derivative of relation (6), we obtain

$$
\dot{\rho}_{D}=\dot{H}(\alpha+2 \beta H) .
$$

Also differentiating (1) with respect to time lead

$$
\dot{H}=-4 \pi G \rho_{D}\left(1+u+w_{D}\right),
$$

where $u=\rho_{m} / \rho_{D}$. Replacing this relation in (7D) and also using the continuity equation (5) we get

$$
\left(1+w_{D}\right)[3 H-4 \pi G(\alpha+2 \beta H)]=4 \pi G(\alpha+2 \beta H) .
$$

Solving the above equation for $w_{D}$ and noting that $u=\frac{\Omega_{m}}{\Omega_{D}}$ as well as

$$
\frac{4 \pi G}{3 H}(\alpha+2 \beta H)=\frac{\Omega_{D}}{2}+\frac{4 \pi G \beta}{3},
$$

we obtain

$$
w_{D}=\frac{\xi-\Omega_{D}}{\Omega_{D}\left(2-\Omega_{D}-\xi\right)},
$$

where $\xi=\frac{8 \pi G \beta}{3}$. It is clear that this relation reduce to its respective one in the GDE when $\xi=0$ [27].

It is easy to see that at late times where $\Omega_{D} \rightarrow 1, w_{D} \rightarrow-1$, which implies that the GGDE in a flat universe mimics a cosmological constant behavior. Also we find that $w_{D}$ of the GDE model cannot cross the phantom divide and the universe has a de Sitter phase at late time.

Next we turn to the deceleration parameter which is defined as

$$
q=-\frac{a \ddot{a}}{\dot{a}^{2}}=-1-\frac{\dot{H}}{H^{2}},
$$

where $a$ is the scale factor. Using Eq. (8) and the definition of $\Omega_{D}$ in (2) we get

$$
\frac{\dot{H}}{H^{2}}=-\frac{3}{2} \Omega_{D}\left(1+u+w_{D}\right) .
$$

Replacing this relation into (12), and using (11) we find

$$
q=\frac{1}{2}-\frac{3}{2} \frac{\xi-\Omega_{D}}{\left(\Omega_{D}+\xi-2\right)} .
$$

One can easily check that the deceleration parameter in GDE is retrieved for $\xi=0$ [27]. At late time where the DE dominates $\left(\Omega_{D} \rightarrow 1\right)$, independent of the value of the $\xi$, we have $q=-1$. Besides, taking $\Omega_{D 0}=0.72$ and adjusting $\xi=0.01$, obtain $q_{0} \approx-0.34$ for the present value of the deceleration parameter which is in agreement with recent observational data [50].

The evolution equation of the GGDE can be obtained as [39]

$$
\frac{d \Omega_{D}}{d \ln a}=-3 \frac{\left(1-\Omega_{D}\right)\left(\xi-\Omega_{D}\right)}{2-\Omega_{D}-\xi} .
$$

\section{B. Stability of the model according to the square sound speed parameter}

The parameter we use through this paper as a factor of stability of the model is the square sound speed $v_{s}^{2}$. In classical theory of perturbation we assume a small fluctuation in the background energy density and we would like to see if the perturbation grows or will collapse. In the linear perturbation regime, the perturbed energy density of the background can be written as

$$
\rho(t, x)=\rho(t)+\delta \rho(t, x)
$$

where $\rho(t)$ is unperturbed background energy density. The energy conservation equation $\left(\nabla_{\mu} T^{\mu \nu}=0\right)$ yields $[4]$

$$
\delta \ddot{\rho}=v_{s}^{2} \nabla^{2} \delta \rho(t, x),
$$




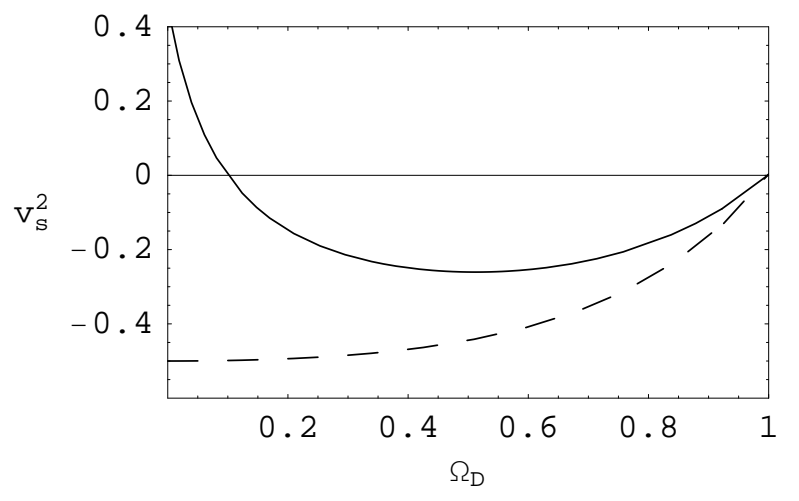

FIG. 1: Evolution of squared sound speed $v_{s}^{2}$ against $\Omega_{D}$ for noninteracting GDE (dashed curve) and GGDE (continues curve) in flat background for $\xi=0.1$.

where $v_{s}^{2}=\frac{d P}{d \rho}$ is the squared of the sound speed. Solutions of equation (17) include two cases of interest. First when $v_{s}^{2}$ is positive Eq. (17) becomes an ordinary wave equation whose solutions would be oscillatory waves of the form $\delta \rho=\delta \rho_{0} e^{-i \omega t+i \vec{k} \cdot \vec{x}}$ which indicates a propagation mode for the density perturbations. The second case of interest occurs when $v_{s}^{2}$ is negative. In this case the frequency of the oscillations becomes pure imaginary and the density perturbations will grow with time as $\delta \rho=\delta \rho_{0} e^{\omega t+i \vec{k} \cdot \vec{x}}$. Thus the growing perturbation with time indicates a possible emergency of instabilities in the background.

Here we would like to obtain the sound speed in a flat FRW background filled with pressureless matter and GGDE while matter and DE components are separately conserved. The definition of the sound speed reads [22]

$$
v_{s}^{2}=\frac{d P}{d \rho}=\frac{\dot{P}}{\dot{\rho}}=\frac{\rho}{\dot{\rho}} \dot{w}+w,
$$

where in the last step we have used $P=w \rho$. Using the conservation equation (5), we obtain

$$
\frac{\rho}{\dot{\rho}}=\frac{-1}{3 H\left(1+w_{D}\right)} .
$$

Also taking the time derivative of Eq. (11) yields

$$
\dot{w}_{D}=\frac{d w}{d \Omega_{D}} \dot{\Omega}_{D}=-\frac{\Omega_{D}\left(2 \xi-\Omega_{D}\right)+\xi(2-\xi)}{\Omega_{D}^{2}\left(2-\Omega_{D}+\xi\right)^{2}} \dot{\Omega}_{D}
$$

Replacing Eqs.(19) and (20) into (18) and also using (15) we get

$$
v_{s}^{2}=-2 \frac{\left(\xi-\Omega_{D}\right)\left(\Omega_{D}-1\right)}{\left(\Omega_{D}+\xi\right)\left(2-\Omega_{D}+\xi\right)^{2}}
$$

where we have used $\frac{d}{d t}=H \frac{d}{d \ln a}$. One can easily see that setting $\xi=0$, this result reduces to its respective relation in [28]. Having the $v_{s}^{2}$ at hand we are ready to discuss about the stability of perturbations. One can easily see from (21) that $v_{s}^{2}$ is negative provided $\xi>\Omega_{D}$. One should note here that $v_{s}^{2}$ can be positive up to present time if $\xi$ is limited to suitable values and thus $\xi$ plays a crucial roles in the stability of perturbations background. However, according to [38], the presented range of parameter $\beta$ does not let us to set $\xi$ to suitable values. This result indicates that due to the negativity of the squared sound speed every small perturbation can grow with time which leads to an instability in the universe. Thus we can not expect a noninteracting GGDE dominated universe in the future as the fate of the universe, however to speak about the stability issue one have to consider other features in the standard theories of perturbation in cosmology but the negativity of the square sound speed can be taken as a sign of instability against small perturbations. The evolution of $v_{s}^{2}$ versus $\Omega_{D}$ is shown in Fig. 1.

\section{STABILITY OF INTERACTING GGDE IN FLAT UNIVERSE}

Based on a traditional and historical manner usually people consider dark matter and DE separately. However, these days observations detect signals of interaction between DM and DE [47]. Also from theoretical point of view 
we know that any conservation law should reflect a symmetry in the Lagrangian and so far we do not know such a symmetry for DE. Thus, it is natural to assume the interaction between the two dark components of the universe.

In order to study the interacting model we consider the energy balance equations as

$$
\begin{aligned}
\dot{\rho}_{m}+3 H \rho_{m} & =Q, \\
\dot{\rho}_{D}+3 H \rho_{D}\left(1+w_{D}\right) & =-Q,
\end{aligned}
$$

where $Q$ represents the interaction term which allows the energy exchange between the two dark components of the universe. The form of $Q$ is a matter of choice and we take it as

$$
Q=3 b^{2} H\left(\rho_{m}+\rho_{D}\right)=3 b^{2} H \rho_{D}(1+u),
$$

with $b^{2}$ being a coupling constant. Taking the above equations into account and following a same steps as the previous section we can find

$$
w_{D}=-\frac{1}{2-\Omega_{D}-\xi}\left(1+\frac{2 b^{2}}{\Omega_{D}}-\frac{\xi}{\Omega_{D}}\right) .
$$

When $b=0, w_{D}$ reduces to its respective relation in the absence of interaction. The first interesting point about the equation of state parameter of the GGDE is that in the interacting case independent of the interaction parameter, $b$, for $0<\xi<1, w_{D}$ can cross the phantom line in the future while $\Omega_{D} \rightarrow 1$.

The deceleration parameter in the presence of an interaction term can be obtained by substituting (25) in (13) and using (12). We find [39]

$$
q=\frac{1}{2}+\frac{3}{2} \frac{\Omega_{D}}{\left(2-\Omega_{D}-\xi\right)}\left(1+\frac{2 b^{2}}{\Omega_{D}}-\frac{\xi}{\Omega_{D}}\right) .
$$

Once again it is clear that setting $b=0$, respective relation in the previous section is retrieved. Finally, we would like to obtain the evolution equation of DE in the presence of interaction. It is a matter of calculation to show that [39]

$$
\frac{d \Omega_{D}}{d \ln a}=3 \Omega_{D}\left[\frac{1-\Omega_{D}}{2-\Omega_{D}-\xi}\left(1+\frac{2 b^{2}}{\Omega_{D}}-\frac{\xi}{\Omega_{D}}\right)-\frac{b^{2}}{\Omega_{D}}\right] .
$$

Now we consider the stability of this model by study the sign of squared sound speed $v_{s}^{2}$. From Eq. (25) we have

$$
\dot{w}_{D}=\frac{d w}{d \Omega_{D}} \dot{\Omega}_{D}=-\frac{\Omega_{D}^{2}+\left(2 b^{2}-\xi\right)\left(2 \Omega_{D}-2+\xi\right)}{\Omega_{D}^{2}\left(2-\Omega_{D}+\xi\right)^{2}} \dot{\Omega}_{D} .
$$

Also from Eqs. (23) and (24) one finds

$$
\frac{\rho}{\dot{\rho}}=-\frac{1}{3 H\left(1+w_{D}+\frac{b^{2}}{\Omega_{D}}\right)}
$$

Taking into account above relations, $\frac{d}{d t}=H \frac{d}{d \ln a}$, and Eq. (27) and replacing these relations into (18) it is a matter of calculation to show that

$$
v_{s}^{2}=-2 \frac{\left(\Omega_{D}-1\right)\left(\xi-\Omega_{D}\right)}{\left(\Omega_{D}-2+\xi\right)^{2}\left(\Omega_{D}+\xi\right)}+2 b^{2} \frac{3 \Omega_{D}-4+\xi}{\left(\Omega_{D}-2+\xi\right)^{2}\left(\Omega_{D}+\xi\right)},
$$

Once again setting $\xi=0$ leads the results of the original GDE in [28]. The evolution of $v_{s}^{2}$ against $\Omega_{D}$ has plotted in Fig. (2) for $b=0.15$ and $\xi=0.1$. This figure reveals that $v_{s}^{2}$ is always negative except for a small period at the beginning of the universe and thus, as the previous case, a background filled with the interacting GGDE seems to be unstable against perturbation. This implies that we cannot obtain a stable GGDE dominated universe. However, the equations show a sensitivity to the value of $\xi$ and we found that it is possible to obtain a stable GGDE dominated universe but it may be in contrast with theoretical values of $\beta$ [38]. One important point is also the sensitivity of the instability to the coupling parameter $b$. The larger $b$, leads to more instability against perturbations. Thus once a gain we find a sign of instability from this model in the presence of the interaction term. 


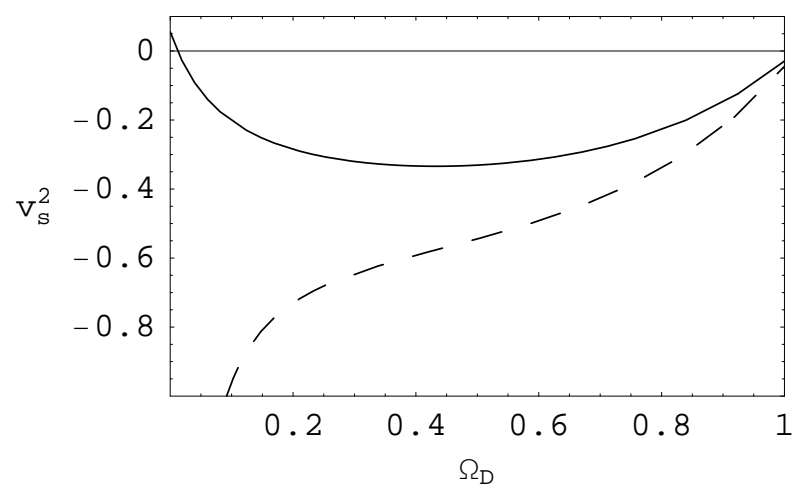

FIG. 2: This figure shows evolution of squared sound speed $v_{s}^{2}$ versus $\Omega_{D}$ for interacting GGDE model. The solid curve corresponds to the GGDE for $b=0.15$ and $\xi=0.1$. Dashed curve is plotted for the original GDE.

\section{STABILITY OF THE INTERACTING GGDE IN NON-FLAT UNIVERSE}

Although it is a general belief that the geometry of the universe is flat, recently this assumption is challenged by several observational evidences. For example, a closed universe is marginally favored by observations from CMB [48]. Besides, the measurements of the cubic correction to the luminosity-distance of supernova measurements support the idea of a closed universe [49]. Thus, there exist enough motivations to consider DE problem in non-flat universe. Here, we would like to extend the study of the GGDE model to a universe with special curvature. In such a case the first Friedmann equations read

$$
H^{2}+\frac{k}{a^{2}}=\frac{1}{3 M_{p}^{2}}\left(\rho_{m}+\rho_{D}\right)
$$

where $k$ is the curvature parameter with $k=-1,0,1$ corresponding to open, flat, and closed universes, respectively. Taking the energy density parameters (2) into account and defining the energy density parameter for the curvature term as $\Omega_{k}=k /\left(a^{2} H^{2}\right)$, the Friedmann equation can be rewritten in the form

$$
1+\Omega_{k}=\Omega_{m}+\Omega_{D}
$$

Using the above equation the energy density ratio becomes

$$
u=\frac{\rho_{m}}{\rho_{D}}=\frac{\Omega_{m}}{\Omega_{D}}=\frac{1+\Omega_{k}-\Omega_{D}}{\Omega_{D}} .
$$

The second Friedmann equation read

$$
\dot{H}=-4 \pi G(P+\rho)+\frac{k}{a^{2}} .
$$

Following the previous sections one can easily see that the equation of state parameter in the interacting non-flat GGDE may be obtained as

$$
w_{D}=-\frac{1}{2-\Omega_{D}-\xi}\left(2-\left(1+\frac{\xi}{\Omega_{D}}\right)\left(1+\frac{\Omega_{k}}{3}\right)+\frac{2 b^{2}}{\Omega_{D}}\left(1+\Omega_{k}\right)\right) .
$$

From the second Friedmann equation, (34), One can easily obtain

$$
\frac{\dot{H}}{H^{2}}=-\Omega_{k}+\frac{3}{2} \Omega_{D}\left[1+u+w_{D}\right]
$$

and therefore the deceleration parameter in this case is obtained as

$$
q=-1-\frac{\dot{H}}{H^{2}}=-1-\Omega_{k}+\frac{3}{2} \Omega_{D}\left[1+u+w_{D}\right]
$$




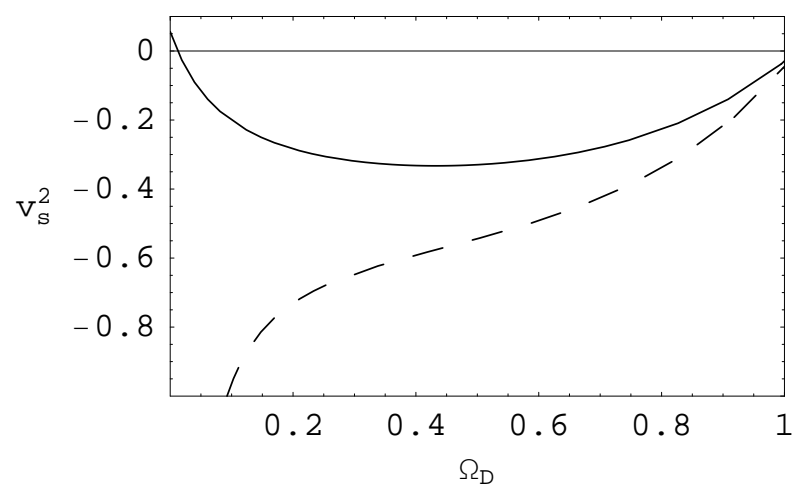

FIG. 3: The evolution of the squared sound speed $v_{s}^{2}$ versus $\Omega_{D}$ for interacting GGDE model in non-flat FRW universe. The solid curve corresponds to the GGDE with $b=0.15$ and $\xi=0.1, \Omega_{k}=0.01$. Dashed curve shows the original GDE model for $b=0.0 .15$.

Substituting Eqs. (33) and (35) in (37) we obtain

$$
q=\frac{1}{2}\left(1+\Omega_{k}\right)-\frac{3 \Omega_{D}}{2\left(2-\Omega_{D}-\xi\right)}\left[2-\left(1+\frac{\xi}{\Omega_{D}}\right)\left(1+\frac{\Omega_{k}}{3}\right)+\frac{2 b^{2}}{\Omega_{D}}\left(1+\Omega_{k}\right)\right] .
$$

Finally the evolution equation of the GGDE in a non-flat interacting case can be written as

$$
\frac{d \Omega_{D}}{d \ln a}=3 \Omega_{D}\left[\frac{\Omega_{k}}{3}+\frac{1-\Omega_{D}}{2-\Omega_{D}-\xi}\left(2-\left(1+\frac{\xi}{\Omega_{D}}\right)\left(1+\frac{\Omega_{k}}{3}\right)+\frac{2 b^{2}}{\Omega_{D}}\left(1+\Omega_{k}\right)\right)-\frac{b^{2}}{\Omega_{D}}\left(1+\Omega_{k}\right)\right] .
$$

In the limiting case $\Omega_{k}=0$, the results of this section, restore their respective equations in flat FRW universe derived in the previous sections and for $\xi=0$ the formulas of [27] are retrieved. The interested reader can see [39] for detailed discussion about this case. Here we consider main task of this section, study the stability of interacting GGDE model in a universe with spacial curvature. From the energy balance equation (23) we can obtain

$$
\frac{\rho}{\dot{\rho}}=-\frac{1}{3 H\left(1+w_{D}+\frac{b^{2}}{\Omega_{D}}\right)} .
$$

Taking the time derivative of Eq. (35), yields

$$
\dot{w}_{D}=-\frac{\Omega_{D}^{2}\left(1-\frac{\Omega_{k}}{3}\right)+\left(2 b^{2}\left(1+\Omega_{k}\right)-\xi\left(1+\frac{\Omega_{k}}{3}\right)\right)\left(2 \Omega_{D}-2+\xi\right)}{\left(2-\Omega_{D}+\xi\right)^{2} \Omega_{D}^{2}} \dot{\Omega}_{D}
$$

Using the above relation, replacing (35) in Eq. (40), and inserting them into (18), after using the time derivative version of (39) one gets

$$
v_{s}^{2}=-2 \frac{\left(\Omega_{D}-1\right)\left(\xi-\Omega_{D}\right)}{\left(\Omega_{D}-2+\xi\right)^{2}\left(\Omega_{D}+\xi\right)}+2 b^{2} \frac{\left(3 \Omega_{D}-4+\xi\right)\left(1+\Omega_{k}\right)}{\left(\Omega_{D}-2+\xi\right)^{2}\left(\Omega_{D}+\xi\right)}-\frac{2}{3} \Omega_{k} \frac{\left(\Omega_{D}+\xi\right)^{2}-\Omega_{D}-3 \xi}{\left(\Omega_{D}-2+\xi\right)^{2}\left(\Omega_{D}+\xi\right)} .
$$

Setting $\Omega_{k}=0=b$ the above relation reduces to the flat noninteracting respective relation. Also the squared sound speed of the flat interacting case can be retrieved when $\Omega_{k}=0$. In order to obtain an insight on the stability issue of the interacting GGDE in a non-flat FRW universe, we should consider the sign of $v_{s}^{2}$ during the evolution of the universe. To this end we plot $v_{s}^{2}$ versus $\Omega_{D}$, where the value of $\Omega_{D}$ indicates different epoches of the universe evolution. Fig. [3] shows the result which clearly indicates an almost same behavior as the flat interacting case and it seems that the presence of the curvature term does not lead to a significant difference in the stability issue at least from this feature (the squared sound speed). The squared sound speed, $\left(v_{s}^{2}\right)$, is positive for $\Omega_{D}<\xi$ and is negative otherwise, indicating the instability of the universe against perturbations in the GGDE background. One can easily find from (42) that increasing $b$ will result more instability in the universe. Finally we can say that the stability of a universe filled with GGDE and matter against small perturbations crucially depends on the value of $\xi$ parameter which is defined on the base of the $\beta$ parameter in Eq. (6). 


\section{SUMMARY AND DISCUSSION}

Due to the lack of observational evidences every DE model which can explain the current acceleration of the universe seems to be acceptable. However, every new model accompanies by many consequences which should be explored. In this paper we considered an important feature, which every DE models should provide. Based on cosmic observations our universe is in a stable DE dominated epoch. In this paper we tried to see if the GGDE model is capable to result in a stable dominated universe. The main point made the authors to consider such an issue in the GGDE comes from the result obtained in [38, 39]. In these papers authors found that the second term in the GGDE energy density (6), has a negative contribution to the energy density. This negativity is in contrast of the first term and we thought may such a behavior could result in some signs of stability in the model. To this end, we used the squared sound speed $\left(v_{s}^{2}=\frac{d P}{d \rho}\right)$ as the main factor for studying the stability. If $v_{s}^{2}$ is positive the GGDE could be stable against perturbations. When $v_{s}^{2}$ is negative we encounter signs of instability in the background spacetime. We have discussed several cases including whether there is or not an interaction between dark matter and GGDE and whether there is or not a curvature term in the background metric. We found that the stability of the GGDE model crucially depends on the parameter $\xi$ and adjusting $\xi$ to suitable values the model is capable to result a stable DE dominated universe. However, observational constraint on the GGDE model [38] rejected mandatory range for $\xi$ to leave a chance of stable GGDE dominated universe as the present state of the universe. As a result, the universe filled with dark matter and GGDE component cannot lead to a stable GGDE dominated universe. We also observed that the instability of the interacting GGDE increases with increasing the interacting coupling parameter $b$. Thus, in comparison to the original GDE, the GGDE just has theoretical chance to lead a GGDE dominated universe, however this is ruled out by the present observational constraints. Readers should note that a complete discussion on the stability issue needs considering different features. Here, we just discussed the sign of the square sound speed in different epoches which can be taken as a sign of stability or instability of the model. Other features of the stability will be addressed elsewhere.

\section{Acknowledgments}

This work has been supported financially by Research Institute for Astronomy and Astrophysics of Maragha (RIAAM) under research project number No. 1/2782-63.

[1] A.G. Riess, et al., Astron. J. 116 (1998) 1009;

S. Perlmutter, et al., Astrophys. J. 517 (1999) 565;

S. Perlmutter, et al., Astrophys. J. 598 (2003) 102;

P. de Bernardis, et al., Nature 404 (2000) 955.

[2] D.N. Spergel et al., Astrophys. J. Suppl. 148, 175 (2003).

[3] D.N. Spergel et al.,Astrophys.J.Suppl.170, 377 (2007).

[4] M. Tegmark et al., Phys. Rev. D 69, 103501 (2004).

[5] M. Tegmark et al., Astrophys. J. 606, 702 (2004).

[6] C. Wetterich, Nucl. Phys B. 302, 668 (1988).

[7] B. Ratra and J. Peebles, Phys. Rev D 37, 321 (1988).

[8] T. Chiba, T. Okabe and M. Yamaguchi, Phys. Rev. D 62, 023511 (2000).

[9] C. Armendariz-Picon, V. Mukhanov, and P. J. Steinhardt, Phys. Rev. Lett. 85, 4438 (2000).

[10] C. Armendariz-Picon, V. Mukhanov, and P. J. Steinhardt, Phys. Rev. D 63, 103510 (2001).

[11] R.R. Caldwell, M. Kamionkowski, N.N. Weinberg, Phys. Rev. Lett. 91, 071301 (2003).

[12] T. Padmanabhan, Phys. Rev. D 66, 021301 (2002);

J. S. Bagla, H. K. Jassal and T. Padmanabhan, Phys. Rev. D 67, 063504 (2003);

L. R. W. Abramo and F. Finelli, Phys. Lett. B 575165 (2003);

J. M. Aguirregabiria and R. Lazkoz, Phys. Rev. D 69, 123502 (2004);

Z. K. Guo and Y. Z. Zhang, JCAP 0408, 010 (2004);

E. J. Copeland, M. R. Garousi, M. Sami and S. Tsujikawa, Phys. Rev. D 71, 043003 (2005).

[13] M. Li, Phys. Lett. B 603, 1 (2004);

D. Pavon, W. Zimdahl, Phys. Lett. B 628 (2005) 206;

B. Wang, Y. Gong and E. Abdalla, Phys. Lett. B 624 (2005) 141;

A. Sheykhi, Class. Quantum Grav. 27 (2010) 025007;

A. Sheykhi, Phys. Lett. B 681 (2009) 205. 
[14] R. G. Cai, Phys. Lett. B 657 (2007) 228.

H. Wei and R. G. Cai, Phys. Lett. B 660 (2008) 113;

A. Sheykhi, Phys. Lett. B 680 (2009) 113;

A. Sheykhi, Phys. Lett. B 682 (2010) 329;

A. Sheykhi, Phys. Rev. D 81 (2010) 023525;

A. Sheykhi, Phys. Lett. B 694 (2011) 284;

K. Karami, et. al., Gen. Rel. Grav. 43 (2011) 27;

M. Jamil and A. Sheykhi, Int. J. Theor. Phys. 50 (2011) 625.

[15] Miao Li, Xiao-Dong Li, Shuang Wang, Yi Wang, arXiv:1103.5870.

[16] S. Capozziello, S. Carloni and A. Troisi, Recent Res. Dev. Astron. Astrophys. 1 (2003) 625;

S. Capozziello, V. F. Cardone, S. Carloni and A. Troisi, Int. J. Mod. Phys. D 12, 1969 (2003).

[17] S. M. Carroll, V. Duvvuri, M. Trodden and M. S. Turner, Phys. Rev. D 70, 043528 (2004).

[18] G. Dvali, G. Gabadadze, M. Porrati, Phys. Lett. B 485, 208 (2000);

M. Carena, J. Lykken, M. Park, J. Santiago, Phys. Rev. D 75 (2007) 026009;

Masato Minamitsuji, Phys. Lett. B 684 (2010) 92.

[19] A. Sheykhi, B. Wang and N. Riazi, Phys. Rev. D 75, 123513 (2007).

[20] F. R. Urban and A. R. Zhitnitsky, Phys. Lett. B 688 (2010) 9; Phys. Rev. D 80 (2009) 063001;

JCAP 0909 (2009) 018;

Nucl. Phys. B 835 (2010) 135.

[21] N. Ohta, Phys. Lett. B 695 (2011) 41, arXiv:1010.1339.

[22] R.G. Cai, Z.L. Tuo, H.B. Zhang, arXiv:1011.3212

[23] K. Kawarabayashi and N. Ohta, Nucl. Phys. B 175, 477 (1980).

[24] E. Witten, Nucl. Phys. B 156 (1979) 269;

G. Veneziano, Nucl. Phys. B 159 (1979) 213.

[25] C. Rosenzweig, J. Schechter and C. G. Trahern, Phys. Rev. D 21 (1980) 3388.

[26] P. Nath and R. L. Arnowitt, Phys. Rev. D 23 (1981) 473.

[27] A. Sheykhi, M.Sadegh Movahed, Gen Relativ Gravit 44 (2012) 449.

[28] E. Ebrahimi and A. Sheykhi, Int. J. Mod. Phys. D20 (2011) 2369.

[29] A. Sheykhi, M. Sadegh Movahed, E. Ebrahimi, Astrophys Space Sci 339 (2012)93.

[30] A. Sheykhi, A. Bagheri, Euro. Phys. Lett., 95 (2011) 39001.

[31] E. Ebrahimi and A. Sheykhi, Phys. Lett. B 706 (2011) 19.

[32] A. Rozas-Fernandez, Phys.Lett. B709 (2012) 313-321.

[33] A. Khodam-Mohammadi, M. Malekjani, M. Monshizadeh, Mod. Phys. Lett. A27, 18 (2012) 1250100.

[34] M. Malekjani, A. Khodam-Mohammadi, arXiv:1202.4154

[35] C.J. Feng, X. Z. Li and X. Y. Shen, arXiv:1105.3253

[36] M. Maggiore, Phys. Rev. D 83, 063514 (2011).

[37] A. R. Zhitnitsky, arXiv:1112.3365.

[38] R. G. Cai, Z. L. Tuo, Y. B. Wu, Y. Y. Zhao, Phys. Rev. D 86 (2012) 023511.

[39] E. Ebrahimi, A. Sheykhi, arXiv:1209.3147,

A. Sheykhi, E. Ebrahimi, Y. Yosefi, arXiv:1210.0781.

[40] K. Karami, M. Mousivand, arXiv:1209.2044.

[41] P. J. E. Peebles and B. Ratra, Rev. Mod. Phys. 75 (2003) 559.

[42] Y. S. Myung, Phys. Lett. B 652 (2007) 223.

[43] K. Y. Kim, H. W. Lee and Y. S. Myung, Phys. Lett. B 660 (2008) 118.

[44] V. Gorini, A. Kamenshchik, U. Moschella, V. Pasquier and A. Starobinsky, Phys. Rev. D 72 (2005) 103518.

[45] H. Sandvik, M. Tegmark, M. Zaldarriaga and I. Waga, Phys. Rev. D 69 (2004) 123524.

[46] Kh. Saaidi, arXiv: 1202.4097.

[47] Bertolami O, Gil Pedro F and Le Delliou M 2007 Phys. Lett. B 654165.

[48] J. L. Sievers, et al., Astrophys. J. 591 (2003) 599;

C.B. Netterfield, et al., Astrophys. J. 571 (2002) 604;

A. Benoit, et al., Astron. Astrophys. 399 (2003) L25;

A. Benoit, et al., Astron. Astrophys. 399 (2003) L19.

[49] R. R. Caldwell, M. Kamionkowski, astro-ph/0403003;

B. Wang, Y. G. Gong, R. K. Su, Phys. Lett. B 605 (2005) 9.

[50] R.A. Daly, et al., J. Astrophys. 677 (2008) 1. 